\title{
Complex absorption hides a Type II QSO in IRAS 13197-1627
}

\author{
M. Dadina and M. Cappi
}

IASF-CNR Sezione di Bologna, via Gobetti 101, 40129 Bologna, Italy

e-mail: dadina@bo.iasf.cnr.it; cappi@bo.iasf.cnr.it

Received 28 July 2003 / Accepted 1 October 2003

\begin{abstract}
We report on the analysis of a BeppoSAX observation of IRAS 13197-1627. The complexity of the broad band $(0.3-100 \mathrm{keV})$ spectrum of the source prevents us from selecting from two possible best-fit models, i.e. one based on a partial covering and the other on a reflection dominated scenario. Whatever the best fit model, the data imply that: 1) the primary spectrum is heavily obscured by at least two absorbers with column densities of the order of $5 \times 10^{23} \mathrm{~cm}^{-2}$ and $\geq 2 \times 10^{24} \mathrm{~cm}^{-2}$; 2) the intrinsic 2-10 keV luminosity of the source is at least $\sim 2 \times 10^{44} \mathrm{erg} \mathrm{s}^{-1}$ thus making IRAS 13197-1627 a type-II QSO (more precisely a type $1.8 \mathrm{QSO}$ ) rather than a Seyfert 1.8 galaxy as previously classified. Furthermore, there is marginal evidence of an absorption line at $E \sim 7.5 \mathrm{keV}$ suggesting a possible outflow with $v \sim 0.1 \mathrm{c}$ similar to recent findings on other QSOs and Seyferts.
\end{abstract}

Key words. galaxies: active - galaxies: individual: IRAS 13197-1627 - X-ray: quasar: general

\section{Introduction}

Much of the research on Active Galactic Nuclei (AGNs) in recent years emphasized the search for Type-II QSOs. Unified models of AGNs (e.g. Antonucci 1993) explain the observed differences between broad (Type-I) and narrow (Type-II) emission-line AGNs as obscuration and viewing angle effects rather than intrinsic physical differences. Although rather successful in describing nearby Seyfert galaxies, unified models seem to fail when extended to their higher-luminosity "relatives", the QSOs (hereinafter, we shall broadly define a Seyfert as having $L_{(2-10 \mathrm{keV})}<10^{44} \mathrm{erg} \mathrm{s}^{-1}$ and a QSO if $L_{(2-10 \mathrm{keV})}>$ $10^{44} \mathrm{erg} \mathrm{s}^{-1}$ ).

On the basis of unified models, the existence of a large population of type-II QSOs, or broadly speaking of a large population of heavily-absorbed high-luminosity AGNs has to be expected. However, because of the intrinsic difficulties to find them, only a limited number of such objects has been identified. Their rarity is somehow embarrassing considering that they are supposed to contribute the most to the total emission of the cosmic X-ray background. This is now supported both observationally from recent Chandra and XMM-Newton deepfields and theoretically from synthesis models of the cosmic X-ray background (see e.g. the recent review by Fabian 2003).

In this paper we report results from the BeppoSAX narrow-field-instruments (Boella et al. 1997a) observation of IRAS 13197-1627. According to these, we find that the source can be identified as the brightest Type-II QSO known to date. Given the above argument, it is thus an interesting case-study

Send offprint requests to: M. Dadina,

e-mail: dadina@bo.iasf.cnr.it to understand the detailed absorption properties of this type of objects.

IRAS 13197-1627, also named MCG-3-34-64 ${ }^{1}$, has been firstly classified as a Seyfert 2 galaxy (Osterbrock \& de Robertis 1985; de Robertis et al. 1988) and, afterward, as a type 1.8 Seyfert by Aguero et al. (1994) and Young et al. (1996). Its basic parameters are given in Table 1.

The morphological classification of its host galaxy is unclear. In optical images it is extended over $\sim 30^{\prime \prime} \times 30^{\prime \prime}$ with only a few indications, if any, of spiral structures (de Robertis et al. 1988). A broad component in the $\mathrm{H} \alpha$ line has been detected in polarized light by Young et al. (1996).

IRAS 13197-1627 was first observed in X-rays by ASCA (Ueno 1995). The ASCA spectrum was extremely steep (the photon index was $\Gamma \sim 3.0 \pm 0.3$ ), with a high absorption column density of $N_{\mathrm{H}} \sim 4 \times 10^{23} \mathrm{~cm}^{-2}$ and with a soft fainter component emerging at energies below $\sim 3 \mathrm{keV}$. ASCA also detected an $\mathrm{FeK} \alpha$ line at $E \sim 6.4 \mathrm{keV}$ with an equivalent width $E W \sim$ $350 \mathrm{eV}$ (Ueno 1995).

The present BeppoSAX dataset has been previously analyzed by Risaliti (2002) in a statistical study of a sample of 20 bright type-II Seyfert galaxies. However, Risaliti (2002) used only a predefined set of 3 spectral models and did not attempt a detailed modeling of all the spectral complexities found in this particular source. The reduced $\chi^{2}$ of the best-fit reported by Risaliti (2002) was $\sim 1.3$, much larger than those reported

1 The source has been often misidentified with MCG-3-34-63, an edge-on galaxy $\sim 2^{\prime}$ north-est of IRAS 13197-1627. We checked that the X-ray emission is indeed coming from MCG-3-34-64 while MCG-3-34-63, is not detected in 0.1-10 keV band. 
Table 1. Basic parameters of IRAS 13197-1627. (1) de Robertis et al. (1988); (2) Aguero et al. (1994); (3) Theureau et al. (1998).

\begin{tabular}{lc}
\hline \hline Hubble Type & S?(1) \\
Seyfert Type & $1.8(2)$ \\
Redshift & $0.01654(3)$ \\
RA (2000) & 132224.462 \\
Dec (2000) & -164342.91 \\
$m_{\mathrm{v}}$ & 14.46 \\
\hline
\end{tabular}

here. The present work thus is complementary to and expands Risaliti's results.

\section{Observation and data reduction}

BeppoSAX observed IRAS 13197-1627 on July 22, 1998. The LECS (Parmar et al. 1997), MECS (Boella et al. 1997b) and PDS (Frontera et al. 1997) exposure times were of 14 ks, 44 ks and $19 \mathrm{ks}$ respectively.

Data reduction has been performed using the standard mission specific software SAXDAS based on the FTOOLS package.

Two sources are visible at about $20^{\prime}$ from the target in the MECS 2-10 keV image. None is detected by the LECS so we can exclude that they contaminate the IRAS 13197-1627 counts at low energies. These sources are also not detected by the MECS in the $5-10 \mathrm{keV}$ energy range. For this reason they should not contribute significantly to the PDS counts. We checked in public archived databases that no other sources with known strong X-ray (2-10 keV) emission are present in the sky field covered by the PDS instrument $\left(1.3^{\circ}\right.$ FWHM, Frontera et al. 1997).

Source counts have been extracted from circular regions centered on IRAS 13197-1627. The LECS and MECS extraction regions have radii of $8^{\prime}$ and $4^{\prime}$ respectively. The LECS, MECS and PDS spectra have been rebinned so as to sample the energy resolution of the instruments using grouping files produced by the ASI Science Data Center (ASDC) and taking care of having at least 20 counts for each obtained energy bin. To subtract the background, we used standard PHA files accumulated from observations of empty sky regions and produced by the BeppoSAX team. We also checked that the results obtained using local backgrounds are consistent with the ones presented here. Most recent calibration files have been used for the spectral analysis.

Figure 1 shows the MECS 2-5 keV (upper panel) and $5-10 \mathrm{keV}$ (middle panel) lightcurves. The source flux is found to be roughly constant during the first half of the observation, while it is significantly (at a confidence level $~ 95 \%$ ) variable by up to $40 \%$ on timescales down to 5-10 ks during the second half. These variations are observed both in the $2-5 \mathrm{keV}$ band where the source spectrum is strongly affected by absorption and in the 5-10 keV energy range where the source should be seen almost directly.

As implied by the hardness ratios shown in Fig. 1 (lower panel), we found no significant spectral variations (with

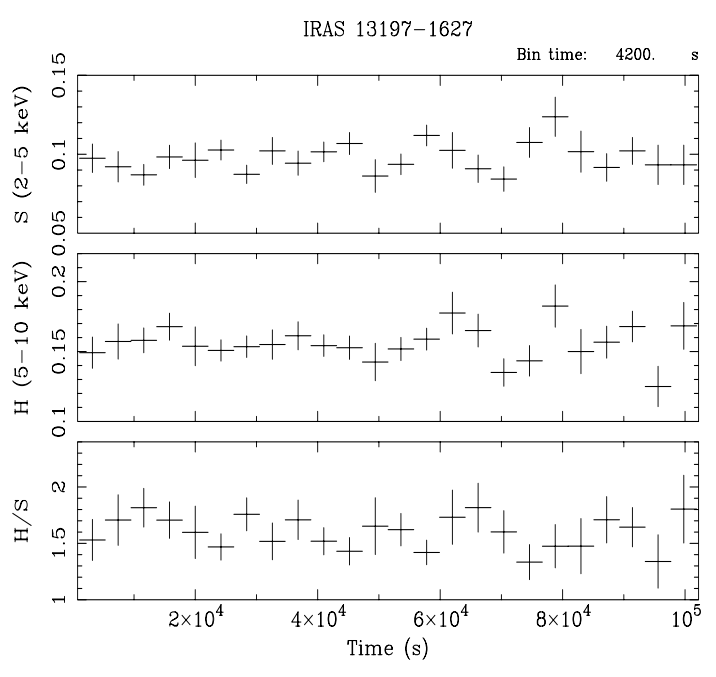

Fig. 1. MECS 2-5 keV (upper panel) 5-10 keV (middle panel) light curves and their ratio as a function of time (lower panel).

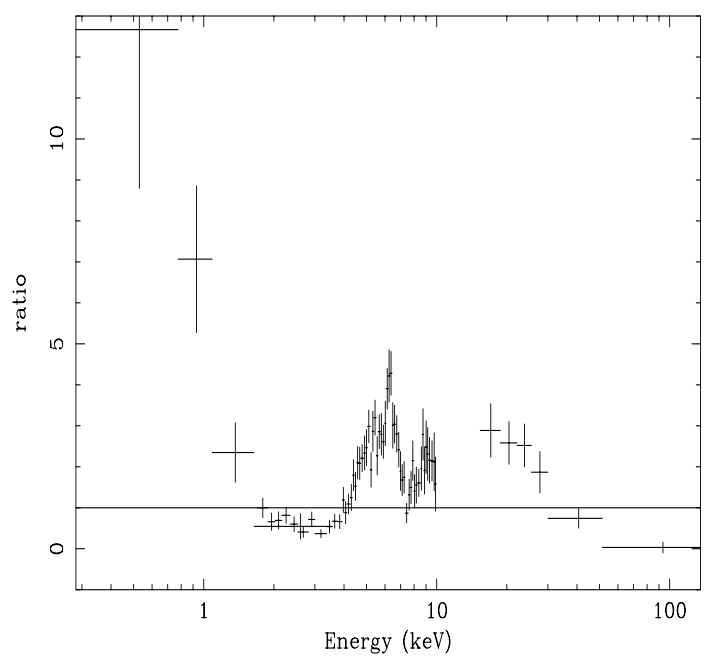

Fig. 2. Data-to-model ratios obtained when fitting the $\sim 0.3-100 \mathrm{keV}$ data with a power-law model with Galactic absorption.

a confidence level $\geq 99 \%$ ) during the observation. We thus performed a time averaged spectral analysis collecting events from the whole period.

\section{Spectral analysis}

As shown by the data-to-model ratios plotted in Fig. 2, the broad-band ( 0.3-100 keV) spectrum of IRAS 13197-1627, once fitted with a simple power-law, clearly shows three main spectral features: 1) a soft excess below $\sim 2-3 \mathrm{keV}$; 2) a lowenergy cut-off due to strong absorption between $\sim 3-5 \mathrm{keV}$; 3 ) a sharp and deep drop of the counts at $E \sim 7 \mathrm{keV}$.

\subsection{Simple spectral parametrization}

To account for the strong absorption and the soft excess we added to the simple power-law model a cold absorber $\left(N_{\mathrm{H}} \sim\right.$ $5 \pm 1 \times 10^{23} \mathrm{~cm}^{-2}$ ) plus a power-law emerging at low energies. In a classical Seyfert 2 spectrum the latter component would be interpreted as due to scattering of the primary emission 
Table 2. SME model. (1) model name; (2) photon index; (3) absorption column density; (4) energy of the absorption edge; (5) optical depth of the absorption edge; (6) $\chi^{2} /$ degrees of freedom.

\begin{tabular}{lccccc}
\hline \hline Model & $\Gamma$ & $\begin{array}{c}N_{\mathrm{H}} \\
10^{22} \mathrm{~cm}^{-2}\end{array}$ & $\begin{array}{c}E_{\text {edge }} \\
\mathrm{keV}\end{array}$ & $\tau_{\text {edge }}$ & $\chi^{2} /$ d.o.f. \\
& $(2)$ & $(3)$ & $(4)$ & $(5)$ & $(6)$ \\
\hline SME & $1.90_{-0.15}^{+0.17}$ & $50.9_{-5.7}^{+6.3}$ & $7.00_{-0.13}^{+0.13}$ & $1.00_{-0.12}^{+0.24}$ & $99.1 / 92$ \\
\hline
\end{tabular}

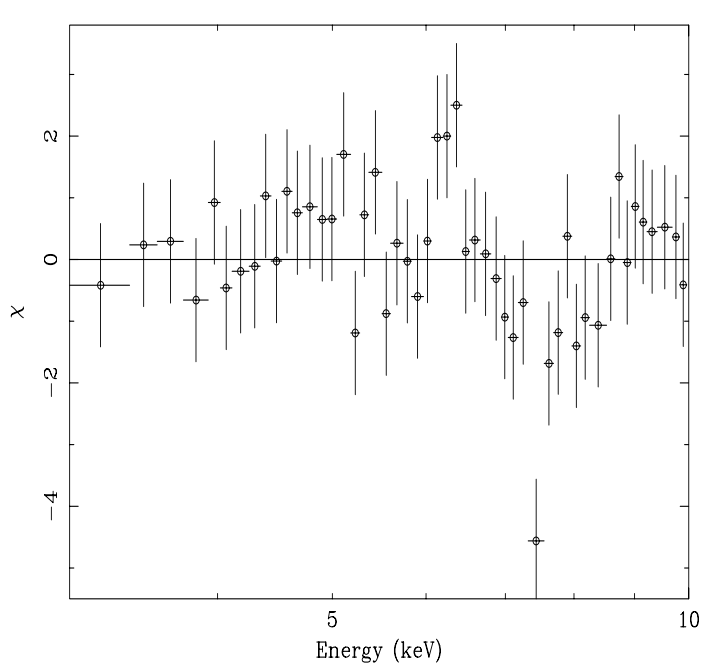

Fig. 3. The 3-10 keV residuals (expressed in terms of standard deviation) for the SM. For clarity, data have been rebinned so as to have a $3 \sigma$ significance for each plotted data point.

(hereinafter we will mention to this model as the "scattering model" SM). The data-to-model residuals (Fig. 3) for SM still show remaining complexities in the $\sim 4-8 \mathrm{keV}$ energy range: an emission feature at $6.4 \mathrm{keV}$, a deep absorption edge at $\sim 7 \mathrm{keV}$ and possible other components arising between $\sim 4-6 \mathrm{keV}$.

To better model the data, we first tried to obtain a simple parametrization of all the features disregarding their physical implication.

The quality of the fit increases drastically after the addition of an absorption edge to the SM $\left(\Delta \chi^{2}=24.3\right.$ for 2 additional parameters, see SME model in Table 2). According to the F-test, this absorption edge is significant at $\geq 99.99 \%$ confidence level (see Fig. 4). The energy of the edge $(E \sim 7.0 \pm$ $0.2 \mathrm{keV}$ ) rules out (at $\geq 99 \%$ ) the presence of iron ionized at levels larger than FeX. If produced by the measured absorption column $\left(N_{\mathrm{H}} \sim 5 \times 10^{23} \mathrm{~cm}^{-2}\right)$ the depth of the edge would require an iron overabundance ranging between 4 and 8 times the solar value.

Moreover, the data-to-model residuals for the SME model still show significant features between $\sim 4$ and $8 \mathrm{keV}$ (see Fig. 5). These features could be fitted by Gaussian emission lines (one broad at $E \sim 4.5 \mathrm{keV}$ and one narrow at $E \sim 6.4 \mathrm{keV}$ ) and/or by Gaussian absorption lines (at $E \sim 6 \mathrm{keV}$ and $E \sim$ $7.5 \mathrm{keV}$ ), all of which significant at more than $90 \%$ confidence.

In summary, the "simple spectral parametrization" demonstrates that a single absorber cannot describe well the data above $\sim 2 \mathrm{keV}$.

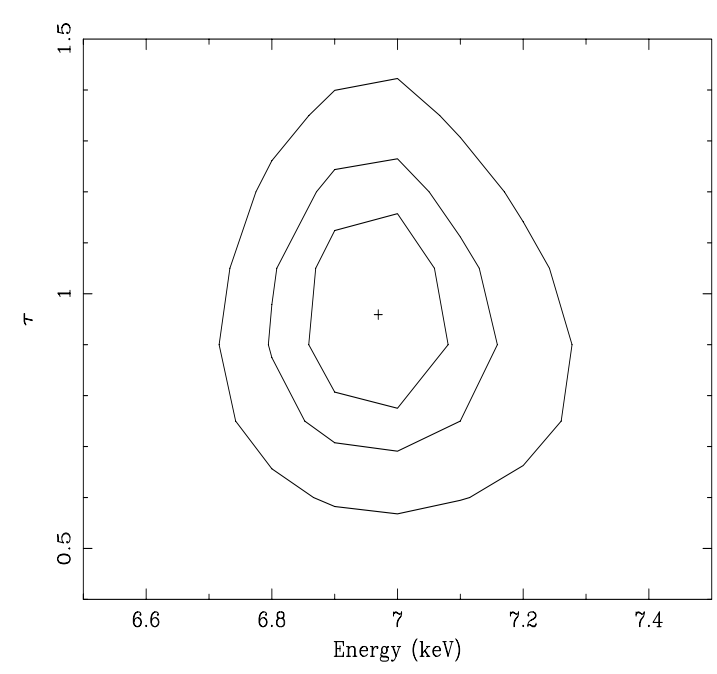

Fig. 4. Confidence contour levels $(68,90$ and $99 \%)$ for the edge optical depth $\tau$ versus its energy centroid.

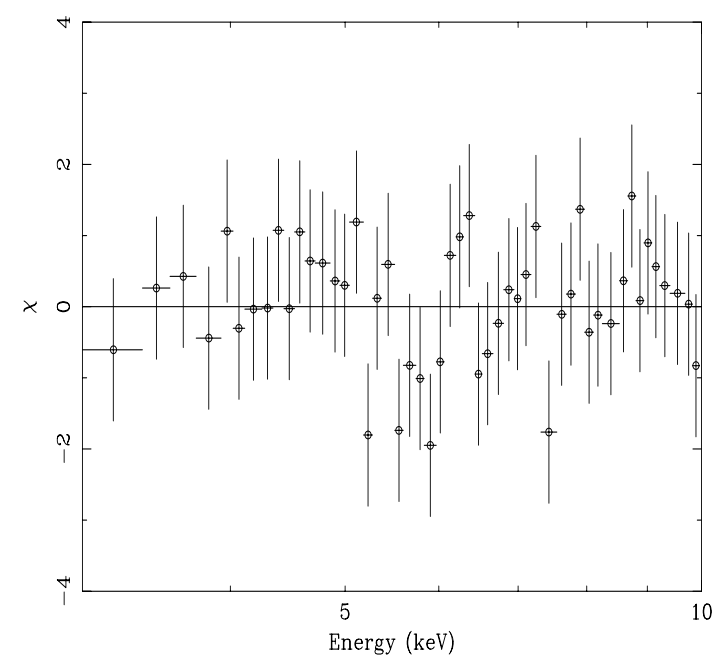

Fig. 5. Residuals in the 3-10 keV energy range for the SME model (data binned as in Fig. 3).

\subsection{Partial covering}

A possible explanation for the deep edge detected at $\sim 7 \mathrm{keV}$ could be the occurrence of an additional heavy absorption that covers only partially the primary emission. This model fits well the data (model PC in Table 3 and Fig. 6) and the parameter values obtained indicate that about $95 \%$ of the primary emission is absorbed by cold matter with $N_{\mathrm{H}} \sim 2 \times 10^{24} \mathrm{~cm}^{-2}$. The photon index of the continuum is quite steep $(\Gamma \sim 3.2 \pm 0.3)$ while the $\mathrm{FeK}_{\alpha}$ line is detected (at a confidence level $\geq 99 \%$ ) 
Table 3. Partial Covering model. (1) model name; (2) photon index; (3) column density of the whole absorber; (4) covering fraction of the partial absorber; (5) column density of the partial absorber; (6) energy of the FeK $\alpha$ emission line; (7) equivalent width $(E W)$ of the FeK $\alpha$ line; (8) $\chi^{2} /$ degrees of freedom.

\begin{tabular}{cccccccc}
\hline \hline Model & $\Gamma$ & $N_{\mathrm{H}, \text { whole }}$ & $C_{\mathrm{f}}$ & $N_{\mathrm{H}, \text { partial }}$ & $E_{\mathrm{FeK} \alpha}$ & $E W$ & $\chi^{2} /$ d.o.f. \\
& & $10^{22} \mathrm{~cm}^{-2}$ & & $10^{22} \mathrm{~cm}^{-2}$ & $\mathrm{keV}$ & $\mathrm{eV}$ & $(8)$ \\
$(1)$ & $(2)$ & $(3)$ & $(4)$ & $(5)$ & $(6)$ & $(7)$ & $82.9 / 90$ \\
\hline PC & $3.17_{-0.15}^{+0.34}$ & $47.8_{-2.1}^{+3.1}$ & $0.94_{-0.03}^{+0.01}$ & $269_{-55}^{+81}$ & $6.42_{-0.12}^{+0.12}$ & $228_{-179}^{+160}$ & 80 \\
\hline
\end{tabular}

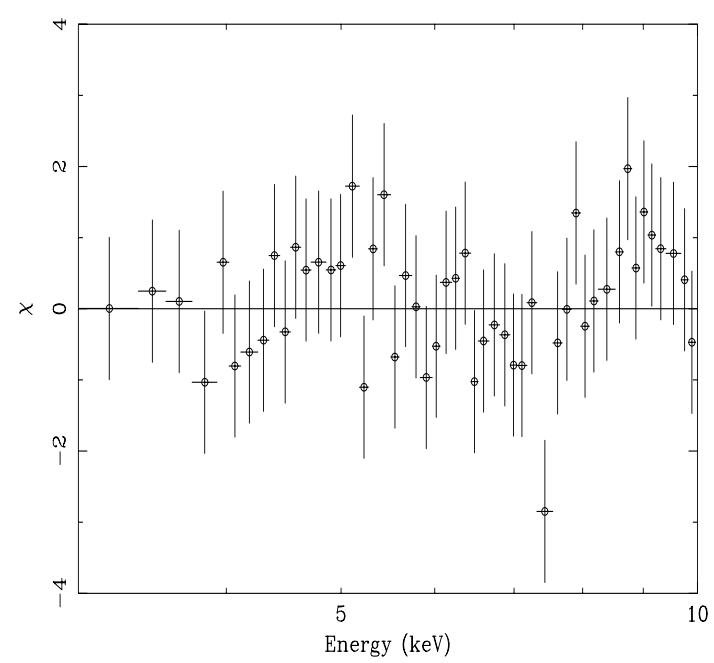

Fig. 6. Data-to-model residuals for the PC model (data binned as in Fig. 3).

at $E \sim 6.4 \pm 0.1 \mathrm{keV}$ with $E W \sim 228 \pm 180 \mathrm{eV}$. With this model the luminosity of the source is found to be $L_{2-10 \mathrm{keV}} \sim$ $2 \times 10^{44} \mathrm{erg} \mathrm{s}^{-1}$. The observed $\mathrm{FeK}_{\alpha}$ line $E W$ is lower than expected for the observed column densities (an absorbing column of $N_{\mathrm{H}} \sim 2 \times 10^{24} \mathrm{~cm}^{-2}$ with a $\sim 95 \%$ coverage plus a $N_{\mathrm{H}} \sim 5 \times$ $10^{23} \mathrm{~cm}^{-2}$ with $100 \%$ coverage should imply an $E W \sim 700 \mathrm{eV}$, Makishima et al. 1986).

\subsection{Pure reflection}

Alternatively to the PC model, the edge at $7 \mathrm{keV}$ could be produced by a reflection component, which dominates the overall spectral shape.

If we add to any of the above mentioned models a cold reflection component (namely PEXRAV model in XSPEC, Magdziarz \& Zdziarski 1995), we obtain that the $R$ parameter that define the relative importance of the reflected continuum with respect to the direct one, reaches very high values $(R \geq$ 10), thus reproducing a completely reflected spectrum.

We thus tried a model where the primary continuum is completely hidden for us and we only see the continuum reflected from the disk plus the associated $\mathrm{FeK} \alpha$ line. This latter component has been parametrized using a relativistic model (the Laor model in XSPEC, see Fabian et al. 2002 for a recent review on this topic). Since the data clearly show a low energy cut-off, we also added an absorption column to the model plus the associated narrow Gaussian emission line. To prevent the parameters of this model to diverge we fixed some values of the reflection

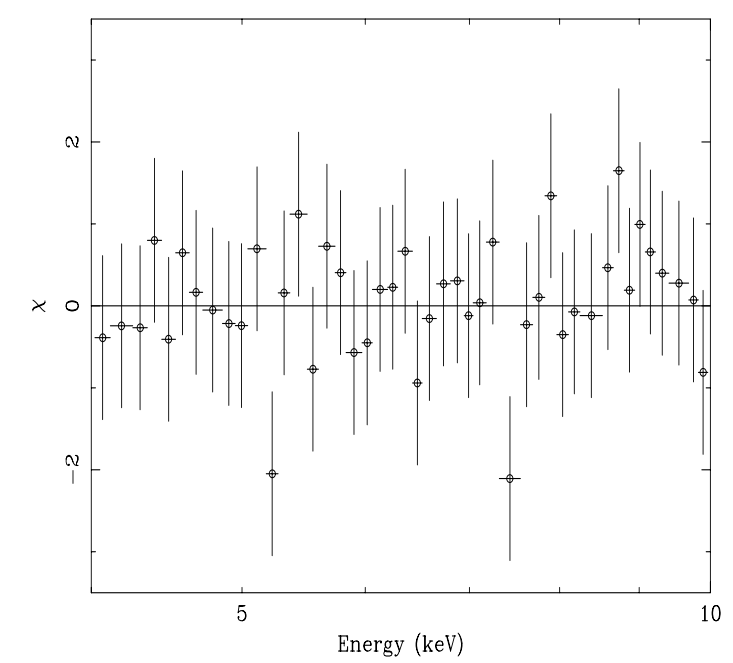

Fig. 7. Data-to-model residuals for the PR model (data binned as in Fig. 3).

component: metal abundance, $R$ and inclination were fixed to solar, 1 and $60^{\circ}$ respectively. The only free parameters of the continuum were thus the power-law photon index, the high energy cut-off and the normalization. Similarly, we fixed many parameters of the relativistic line as follows: line energy $E=$ $6.4 \mathrm{keV}$, emissivity index $i=-3$, inclination angle $=60^{\circ}$, internal radius of the emitting region $=1.25$ gravitational radii $r_{\mathrm{g}}$. The only free parameters of the Laor model thus were the external radius of the line emitting region $\left(r_{\text {out }}\right)$ and normalization.

This model well describes the data (PR model in Table 4 and Fig. 7) and the inferred intrinsic luminosity of the source is found to be $L_{2-10 \mathrm{keV}} \geq 2 \times 10^{44} \mathrm{erg} \mathrm{s}^{-1}$. The fit results do not depend on the energy of the relativistic line. In fact, values of $E_{\text {Laor }}$ from 6.4 to $6.9 \mathrm{keV}$ give fits of the same quality $\left(\Delta \chi^{2} \leq 0.2\right)$. The $E W$ of the relativistic line is rather large $(E W \sim 3.8 \mathrm{keV})$ and it is in agreement with what is expected from a pure reflection spectrum (Matt et al. 1996). The outer radius of the line emitting region is found to be $\sim 5-7 r_{\mathrm{g}}$, a rather extreme value. The energy and $E W$ of the narrow line are in perfect agreement with what expected for a line produced via transmission in a cold absorber with the measured $N_{\mathrm{H}}$ of $\sim 4 \times$ $10^{23} \mathrm{~cm}^{-2}$ (Makishima 1986).

\subsection{An absorption line at $7.5 \mathrm{keV}$ ?}

In the data-to-model residuals both for the PC and PR model there is a marginal evidence of an additional absorption 
Table 4. Pure Reflection model. (1) model name; (2) photon index; (3) absorption column density; (4) cut-off energy; (5) energy of the relativistic FeK $\alpha$ line; (6) external radius of the region of emission of the relativistic line expressed in units of gravitational radii; (7) equivalent width of the relativistic line; (8) energy of the narrow FeK $\alpha$ line; (9) equivalent width of the narrow line; (10) $\chi^{2} /$ degrees of freedom. ${ }^{p}$ pegged parameter. ${ }^{f}$ fixed parameter.

\begin{tabular}{|c|c|c|c|c|c|c|c|c|c|}
\hline Model & $\Gamma$ & $\begin{array}{c}N_{\mathrm{H}} \\
10^{22} \mathrm{~cm}^{-2}\end{array}$ & $\begin{array}{c}E_{\text {cut-off }} \\
\mathrm{keV}\end{array}$ & $\begin{array}{l}E_{\text {Laor }} \\
\mathrm{keV}\end{array}$ & $\begin{array}{c}r_{\text {out }} \\
\mathrm{keV}\end{array}$ & $\begin{array}{c}E W_{\text {Laor }} \\
\mathrm{keV}\end{array}$ & $\begin{array}{l}E_{\text {narr. }} \\
\mathrm{keV}\end{array}$ & $\begin{array}{c}E W_{\text {narr. }} \\
\mathrm{eV}\end{array}$ & $\chi^{2} /$ d.o.f. \\
\hline (1) & (2) & (3) & (4) & (5) & (6) & (7) & (8) & (9) & (10) \\
\hline PR & $2.30_{-0.31}^{+0.29}$ & $41.2_{-10.8}^{+11.5}$ & $76_{-42}^{+435, p}$ & $6.40^{f}$ & $6.05_{-0.83}^{+0.84}$ & $3.81_{-1.9}^{+4.1}$ & $6.40_{-0.10}^{+0.10}$ & $356_{-143}^{+186}$ & $66.8 / 88$ \\
\hline
\end{tabular}

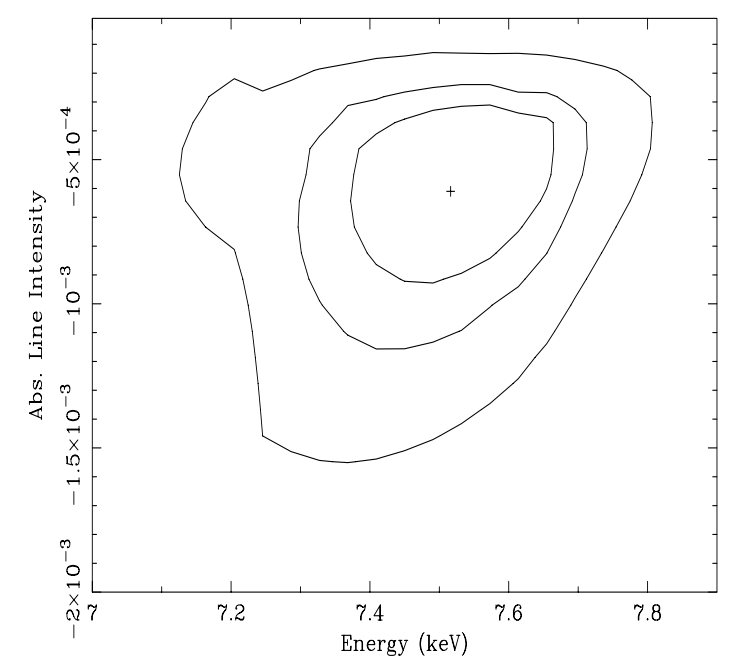

Fig. 8. Confidence contour levels for the absorption line at $\sim 7.5 \mathrm{keV}$ in the PC model.

feature at $E \sim 7.5 \mathrm{keV}$. We checked this hypothesis by adding an absorption Gaussian line to the two models.

The quality of the fit drastically increases in the case of the partial covering $\left(\Delta \chi^{2}=15.2\right.$ for 2 additional parameters, Fig. 8) while the feature seems to be marginal adopting the PR model as baseline $\left(\Delta \chi^{2}=5.4\right)$.

The feature is found to be at $E \sim 7.5 \pm 0.2 \mathrm{keV}$ and to be narrow $(\sigma<0.47 \mathrm{keV})$ while its $E W$ is $\sim-330 \pm 180 \mathrm{eV}$. Its narrowness, in particular, seems to exclude that it could be associated to an unresolved additional edge due to warm matter.

This absorption line could be associated with resonant absorption due to He-like and/or H-like Fe. We tested this hypothesis modeling the feature with the SIABS model (Kinkhabwala et al. 2003) assuming it is due to He-like Fe. We obtained a good quality fit with physically plausible parameters, i.e. $N_{\mathrm{Fe}} \sim$ $10^{19} \mathrm{~cm}^{-2}$, outward velocity $v \sim 0.11 \mathrm{c}$ and dispersion $\Delta v \sim$ $4500 \mathrm{~km} \mathrm{~s}^{-1}$.

\section{Discussion}

The overall 0.3-100 keV spectrum of IRAS 1319-1627 is dominated by cold absorption and by an extra soft component that rises below $\sim 3 \mathrm{keV}$. This soft component is well modeled by a power-law with the same photon index of the hard component. Thus, as a first approximation, this spectrum appears as a very common (i.e. highly absorbed) X-ray spectrum of a Seyfert 2 galaxy where the low energy band is dominated by a scattered component (Smith \& Done 1996; Turner et al. 1997; Risaliti 2002).

However, unlike other spectra of known Seyfert 2s, there is a major unexpected feature in the spectrum of IRAS 13197-1627 that is a very deep absorption edge at $\sim 7 \mathrm{keV}$. The edge energy is consistent with it being produced in a cold medium and rules out the presence of any warm material. The depth of the edge requires an absorber with $N_{\mathrm{H}} \sim$ $2 \times 10^{24} \mathrm{~cm}^{-2}$, i.e. $\sim 3-4$ times larger than what found by modeling the low energy cut-off with a single absorption column. Thus, to explain this edge, we are forced to invoke either that the source is partially covered or that it is completely obscured and its spectrum is dominated by the reflected component.

\subsection{The pure reflection and partial covering scenarios}

The pure reflection scenario seems rather extreme since a strong relativistic line $(E W \sim 3.8 \mathrm{keV})$ produced within $\sim 7$ gravitational radii is required to fit the data. Such an extreme line recalls the one that has been recently invoked for the narrow line Seyfert 1 MCG-6-30-15 (Wilms et al. 2001).

Bassani et al. (1999) suggested to use the ratio $R_{\mathrm{CT}}$ between the absorption corrected OIII[5007 $\AA$ ] flux and the observed 2-10 keV flux to identify Compton-thick sources. In their scheme, Compton-thick sources have $R_{\mathrm{CT}} \leq 0.1$. In the present case the $2-10 \mathrm{keV}$ flux is $\sim 4 \times 10^{-12} \mathrm{erg} \mathrm{s}^{-1} \mathrm{~cm}^{-2}$ and the absorption corrected $\mathrm{F}[5077 \AA]$ flux is $\sim 4 \times 10^{-12} \mathrm{erg} \mathrm{cm}^{-2} \mathrm{~s}^{-1}$ (Young et al. 1996) yielding $R_{\mathrm{CT}} \sim 1$. Also using the ASCA flux (Ueno 1995) one obtains $R_{\mathrm{CT}} \sim 0.5$. Therefore, according to this criterium, IRAS 13197-1627 should not be a Comptonthick source, making the pure reflection scenario even more implausible.

The partial covering scenario appears to be more likely not last for its simplicity. Nevertheless also in this case there are difficulties in interpreting the best fit parameters. First of all, the best fit photon index $\Gamma \sim 3$ is rather steep for Seyfert galaxies (Smith \& Done 1996; Turner et al. 1997) and it is only marginally consistent with what found for the most extreme Narrow Lines Seyfert 1 (NLSY1, Boller et al. 1996; Vaughan et al. 1999), i.e. the radio quiet AGNs with the known steeper spectra. It is worth noting here that another possible similitude with this class of sources is the observed variability $(\sim 40 \%)$ down to timescales of $\sim 5-10 \mathrm{ks}$. Another inconsistency in the partial covering scenario is that the $E W$ of the FeK $\alpha$ line is lower than what expected for the observed absorption (see Sect. 3.2). 
Present results show interesting analogies with what found for NLSY1s. This class of objects are generally characterized in X-rays by a strong soft excess, a steep power-law and extreme variability (Boller et al. 1996; Brandt et al. 1997; Brandt \& Boller 1998; Vaughan et al. 1999). Recent XMM-Newton results have added to this list, a new "typical" feature: a sharp spectral drop above $7 \mathrm{keV}$ (Boller et al. 2001, 2003; Pounds et al. 2003a). Apart from the soft X-rays (that are here absorbed), IRAS 13197-1627 shows all the above mentioned characteristics of NLSY1s. The spectral complexity measured in IRAS 13197-1627 is indeed very similar to what recently observed in MKN 766 (Pounds et al. 2003a), 1H 0707-495 (Boller et al. 2002) and IRAS 13224-38098 (Boller et al. 2003a) where also in these cases the authors were left with two possible scenarios that described the data equally well: either a reflection dominated or a partial covering scenario.

A theoretical explanation for the reflection dominated scenario has been recently proposed by Fabian et al. (2002). The main assumption of this model is that, in high accretion rate systems like NLSY1s, instabilities in the accretion may lead to the formation of multiple reflectors in the form of cold, dense material clumped into deep sheets or rings.

Within the partial covering scenario, it could be that, as proposed by Pounds et al. (2003a) for MKN 766, dense ionized clouds or ejecta close to the super-massive black hole are responsible for the deep edge observed with XMM-Newton. Also in this case our data are in good agreement with the model with the exception of the ionization state of the partially absorbing matter, our data requiring near-neutral $\mathrm{Fe}$.

To disentangle between these two hypothesis better quality data above $3 \mathrm{keV}$ (e.g. from XMM-Newton) are needed. Nevertheless, it is worth noting here that both scenarios imply that the intrinsic $2-10 \mathrm{keV}$ luminosity of the source is $\geq 2 \times$ $10^{44} \mathrm{erg} \mathrm{s}^{-1}$. This makes IRAS 13197-1627 the nearest and brightest type-1.8 QSO known to-date. This source thus offers a unique possibility to study the spectral properties of this class of objects and to test unified models at the bright end of the luminosity function of Seyfert galaxies. This is of particular importance given that these objects are thought to contribute the most of the cosmic X-ray background. IRAS 13197-1627 is thus an important case study to understand what the absorption characteristics of luminous QSO could be.

\subsection{Possible origin of the complex absorption}

As stated above, the spectral analysis shows that the source has heavy and complex absorption that cannot be modeled by a single absorber.

We speculate that the most plausible scenario is the one in which the lower column is due to the putative dusty torus while the heavier absorption is due to dense clouds, blobs or winds close to the X-ray emitting region. The presence of a dusty torus, in fact, would explain the different columns measured in X-rays $\left(N_{\mathrm{H}} \sim 5 \times 10^{23} \mathrm{~cm}^{-2}\right)$ and in optical (the $E_{B-V}$ in the direction of the nucleus implies a $N_{\mathrm{H}} \sim 1.5 \times 10^{21} \mathrm{~cm}^{-2}$, Lumsden et al. 2001). On the other hand, dense blobs or clouds close to the X-ray source may either partially cover or block the nuclear emission without obscuring the component reflected by the accretion disk.

It is worth noting here that this picture would also be able to explain, if confirmed, the absorption feature at $\sim 7.5 \mathrm{keV}$. This, in fact, could be interpreted as He- or H-like Fe resonant absorption by outflowing matter. The inferred velocity of this outflow then should be $\sim 0.1 \mathrm{c}$, similar to what found in PG1211+143 (Pounds et al. 2003b).

\section{Conclusion}

The BeppoSAX X-ray spectrum of IRAS $13197-1627$ is so complex that we could not find a single best-fit model and were left with two possible scenarios: $i$ ) one where the primary emission is completely blocked and the resulting spectrum is completely reflection dominated and ii) one where the spectrum is due to a partial covering of the X-ray source.

Despite this ambiguity, a firm conclusion of this work is that any modeling of the X-ray spectrum implies an intrinsic luminosity of at least $L_{2-10 \mathrm{keV}} \sim 2.0 \times 10^{44} \mathrm{erg} \mathrm{s}^{-1}$ thus making IRAS 13197-1627 the nearest and brightest example of a type-II QSO.

Moreover, both scenarios require a complex absorption structure composed of at least two heavy absorbers. We speculate that these could be a dusty torus and clouds, blobs or winds closer to the nucleus. Finally, if the absorption line marginally detected at $7.5 \mathrm{keV}$ is identified with resonant absorption from $\mathrm{He}$ - or $\mathrm{H}$-like $\mathrm{Fe}$, then we infer that some of matter (e.g. the clouds or the wind) should have an outward velocity of the order of $\sim 0.1 \mathrm{c}$.

Acknowledgements. We are very grateful to G. G. C. Palumbo and L. Foschini for useful discussions. M.D. gratefully acknowledge the Italian Space Agency (ASI) for financial support under contract $\mathrm{I} / \mathrm{R} / 042 / 02$. This research has made use of $\mathrm{t}$ he ASDC (ASI Science Data Center) database.

\section{References}

Aguero, E. L., Calderon, J. H., Paolantonio, S., \& Suarez Boedo, E. 1994, PASP, 106, 978

Antonucci, R. R. J. 1993, ARA\&A, 31, 473

Bassani, L., Dadina, M., Maiolino, R., et al. 1999, ApJS, 121, 473

Boella, G., Butler, R. C., Perola, G. C., et al. 1997a, A\&AS, 122, 299

Boella, G., Chiappetti, L., Conti, G., et al. 1997b, A\&AS, 122, 299

Boller, T., Brandt, W. N., \& Fink, H. 1996, A\&A, 305, 53

Boller, T., Fabian, A. C., Sunyaev, R., et al. 2002, MNRAS, 329, L1

Boller, T., Tanaka, Y., \& Fabian, A. C., MNRAS, accepted [astro-ph/0305239]

Brandt, W. N., \& Boller, T. 1998, Accretion Processes in Astrophysical Systems: Some Like it Hot!, 191

Brandt, W. N., Mathur, S., \& Elvis, M. 1997, MNRAS, 285, L25

Carter, D. 1984, Astron. Express, 1, 61

De Grijp, M. H. K., Miley, G. K., \& Lub, J. 1987, A\&AS, 70, 95

de Robertis, M. M., Hutchings, J. B., \& Pitts, R. E. 1988, AJ, 95, 1371

Fabian, A. C., Ballantyne, D. R., Merloni, A., et al. 2002, MNRAS, 331, L35

Frontera, F., Costa, E., Dal Fiume, D., et al. 1997, A\&AS, 122, 357

Guilbert, P. W., \& Rees, M. J. 1988, MNRAS, 233, 475

Ghisellini, G., Haardt, F., \& Matt, G. 1994, MNRAS, 267, 743 
Lightman, A. P., \& White, T. R. 1988, ApJ, 335, 57

Lumsden, S. L., Heisler, C. A., Bailey, J. A., Hough, J. H., \& Young, S. 2001, MNRAS, 327, 459

Kinkhabwala, A., Behar, E., Sako, M., et al. 2003, ApJ, submitted [astro-ph/0304332]

Magdziarz, P., \& Zdziarski, A. A. 1995, MNRAS, 273, 837

Matt, G., Brandt, W. N., \& Fabian, A. C. 1996, MNRAS, 280, 823

Osterbrock, D. E., \& de Robertis, M. M. 1985, PASP, 97, 1129

Parmar, A. N., Martin, D. D. E., Bavdaz, M., et al. 1997, A\&AS, 122, 309

Pounds, K. A., Reeves, J. N., Page, K. L., Wynn, G. A., \& O'Brien, P. T. 2003a, MNRAS, 342, 1147

Pounds, K. A., Reeves, J. N., King, A. R., et al. 2003b, MNRAS, 345, 705
Risaliti, G. 2002, A\&A, 386, 379

Smith, D. A., \& Done, C. 1996, MNRAS, 280, 355

Theureau, G., Rauzy, S., Bottinelli, L., \& Gouguenheim, L. 1998, A\&A, 340, 21

Turner, T. J., George, I. M., Nandra, K., \& Mushotzky, R. F. 1997, ApJS, 113, 23

Vaughan, S., Reeves, J., Warwick, R., \& Edelson, R. 1999, MNRAS, 309, 113

Wilms, J., Reynolds, C. S., Begelman, M. C., et al. 2001, MNRAS, 328, L27

Young, S., Hough, J. H., Efstathiou, A., et al. 1996, MNRAS, 281, 1206

Ueno, S. 1995, Ph.D. Thesis 